\title{
Comments on the Article "Improved Hemodynamics with the Use of Prophylactic Infusion of Epinephrine and or Norepinephrine during Transcatheter Aortic Valve Replacement (TAVR)"
}

\author{
Carlos Galhardo Jr. ${ }^{1}$, Carlos Darcy Alves Bersot ${ }^{2}$ \\ ${ }^{1}$ Departament of Anesthesia at Unimed-Rio Hospital, Rio de Janeiro, Brazil \\ ${ }^{2}$ Departament of Anesthesia at Lagoa Federal Hospital-SUS, Rio de Janeiro, Brazil \\ Email: carlosbersot@gmail.com
}

Received 29 June 2015; accepted 26 October 2015; published 29 October 2015

Copyright (C) 2015 by authors and Scientific Research Publishing Inc.

This work is licensed under the Creative Commons Attribution International License (CC BY). http://creativecommons.org/licenses/by/4.0/

\section{(c) (i) Open Access}

\begin{abstract}
Transcatheter aortic valve replacement (TAVR) is a new therapeutic option to treat patients with symptomatic severe aortic stenosis, and a prohibitive mortality risk for conventional surgical AVR2. A thorough understanding of the procedural steps involved in TAVR is imperative for all components of the heart time. Balloon aortic valvuloplasty under rapid ventricular pacing is one of the main procedural steps, and is usually performed before valve insertion to enable passage of the prosthesis through the stenotic native aortic valve. Hemodynamic collapse necessitating prompt intervention may occur at different stages during the procedure.
\end{abstract}

\section{Keywords}

\section{Cardiovascular, Anesthesia, Surgery, Hemodynamics Control}

We read with interest the article entitled "Improved Hemodynamics with the Use of Prophylactic Infusion of Epinephrine and/or Norepinephrine during Transcatheter Aortic Valve Replacement (TAVR)” by Berthune W. and colleagues [1]. The authors reported that prophylatic infusion of vasoactive agents in patients undergoing TAVR was related to improved hemodynamic recovery after rapid ventricular pacing stimulation. We would like to comment on the results and why this strategy is useful and frequently accepted in different centers. 
Patients with severe aortic stenosis (AS) often have little myocardial reserve, especially in the presence of hypertrophied LV, coronary artery disease, or LV systolic and diastolic dysfunction. Tachycardia and hypotension should be avoided in this group of patients because of high risk of myocardial ischemia, arrhythmias and hemodynamic collapse.

Transcatheter aortic valve replacement (TAVR) is a new therapeutic option to treat patient with symptomatic severe aortic stenosis, and a prohibitive mortality risk for conventional surgical AVR [2]. A thorough understanding of the procedural steps involved in TAVR is imperative for all components of the heart time. Balloon aortic valvuloplasty under rapid ventricular pacing is one of the main procedural steps, and is usually performed before valve insertion to enable passage of the prosthesis through the stenotic native aortic valve. Hemodynamic collapse necessitating prompt intervention may occur at different stages during the procedure.

When performing balloon aortic valvuloplasty or during deployment of a balloon-expandable valve (Edwards Sapien), rapid ventricular pacing is used to decrease cardiac output, transvalvular flow, and cardiac motion, thus reducing the likelihood of device dislodgment. The heart may take some time to recover from this insult, and with that, vasopressor and inotropic support may be required after rapid ventricular pacing. A particular advantage of this method is the near immediate onset and offset of action. Serious complications associated with temporary cardiac pacing of short duration are rare [3].

There are some differential diagnosis of prolonged hypotension in patient undergoing TAVR that we need to be aware, such as: hypovolemia, arrhythmias, excessive bleeding from vascular access or cardiac tamponade. Therefore, management of volume depleted patient as well as maintenance of mean arterial pressure of $>75$ mmHg prior to initiation of rapid ventricular pacing are important strategies to avoid complications [4]. Fassl et al. [5] also increase the blood pressure before starting RVP and have a goal of $75 \mathrm{~mm} \mathrm{Hg}$ of MAP for transapical aortic valve implantation. Infusions or boluses of vasopressors (epinephrine, norepinephrine, phenylephrine and vasopressin) may be required to maintain hemodynamic stability during the case. In the retrospective review by Bethune W. et al. [1], they demonstrated that myocardial recovery after RVP was superior with preemptive continuous infusion of either epinephrine or norepinephrine, avoiding swings in blood pressure. Although this strategy seems to be more beneficial further studies need to address if short periods of hypotension after RVP have any impact on patient outcome after transcatheter aortic valve replacement.

\section{References}

[1] Bethune, W., Konstadt, S., Trunfio, G., Belliveau, L., Kronenfeld, M., Keilin, C. and Feierman, D.E. (2015) Improved Hemodynamics with the Use of Prophylactic Infusion of Epinephrine and/or Norepinephrine during Transcatheter Aortic Valve Replacement (TAVR). Open Journal of Anesthesiology, 5, 130-134. http://dx.doi.org/10.4236/ojanes.2015.56024

[2] Nishimura, R.A., Otto, C.M., Bonow, R.O., et al. (2014) AHA/ACC Guideline for the Management of Patient with Valvular Heart Disease. Journal of the American College of Cardiology, 63, e57-e185. http://dx.doi.org/10.1016/j.jacc.2014.02.536

[3] Murphy, J.J. (1996) Current Practice: Complications of Temporary Transvenous Cardiac Pacing. BMJ, 312, 1134. http://dx.doi.org/10.1136/bmj.312.7039.1134

[4] Holmes Jr., D.R., Mack, M.J., Kaul, S., et al. (2012) ACCF/AATS/SCAI/STS Expert Consensus Document on Transcatheter Aortic Valve Replacement. The Journal of Thoracic and Cardiovascular Surgery, 144, e29-e84. http://dx.doi.org/10.1016/j.jtcvs.2012.03.001

[5] Fassl, J., Walther, T., Groesdonk, H.V., et al. (2009) Anesthesia Management for Transapical Transcatheter Aortic Valve Implantation: A Case Series. Journal of Cardiothoracic and Vascular Anesthesia, 23, 286-291. http://dx.doi.org/10.1053/j.jvca.2008.12.026 